\title{
RADIATION PERFORMANCES OF A DIPOLE ARRAY CONFIGURATION INSERTED IN A LEFT-HANDED MEDIA
}

\author{
E. Sáenz ${ }^{(1)}$, R. Gonzalo ${ }^{(1)}$, I. Ederra $^{(1)}$, P. De Maagt ${ }^{(2)}$ \\ ${ }^{(1)}$ Electrical and Electronic Engineering Department, Universidad Pública de Navarra \\ Campus Arrosadia, E-31006, Pamplona, Navarra, Spain. \\ E-mail: ramon@unavarra.es \\ (2) European Space Research and Technology Centre, ESTEC, \\ PO Box 299, 2201 AG Noordwijk, The Netherlands \\ E-mail: Peter.de.Maagt@esa.int
}

\begin{abstract}
In this work, the radiation improvements achieved when Left Handed Media (LHM) are combined with dipole antennas has been analysed. The behaviour of two dipole antennas in array configuration when a Left Handed superstrate and substrate are placed over and under them has been analysed. Different positions of the dipoles have been studied. Besides, dielectric losses and copper SRRs and CLSs have been taken into account for different distance between dipoles in order to analysed their influence in the radiation properties. Simulation results are presented showing high efficiency antennas with enhanced gain patterns.
\end{abstract}

\section{INTRODUCTION}

Nowadays, the study of the so-called metamaterials, i.e, materials with electromagnetic properties that initially can not be readily found in nature, has found increasing interest. One of the fields that has experimented a higher development has been the one of the Left Handed Materials (LHM). LHMs denote artificially constructed materials characterized for exhibiting simultaneously negative values of electric permittivity $(\varepsilon)$ as well as magnetic permeability $(\mu)$. As a result, the refraction index is also negative, having extraordinary properties that were firstly described by Veselago in 1968 [1].

In the last years, different LHM structures have been proposed and analysed. In this case, a unit cell designed by Professor Ziolkowski has been used [2]. The LHM-media is based on a substrate with embedded capacitively loaded strips (CLSs) that produce a strong dielectric-like response, and split ring resonators (SRRs), that produce a strong magnetic material-like response [2]. These LHM-medias were formed by unit cells which were characterised with ANSOFT's High Frequency Structure Simulator (HFSS). That configuration can be understood as a resonator with pass band properties, acting as a LHM at the X band. From Maxwell's equations, these types of structures can be physically scaled in order to tune the operational band of frequencies at which they act as a LHM.

Knowing the behaviour of these unit cells and the response of a single dipole with a LHM superstrate over it [3], this paper will analyse the radiation properties of a lineal array formed by two dipole antennas and a LHM media. Besides, the effect of losses in the dielectric and the use of copper as metallic strips will be analysed. High efficiency antennas with enhanced gain patterns are obtained.

\section{ARRAY CONFIGURATION}

The behaviour of an array made up of two identical dipoles and a LHM media will be analysed. Both dipole antennas are inside the LHM media, what is constituted by two LHM structures. One of them is placed over the dipoles (superstrate) and the other one is under the dipoles (substrate). The superstrate and substrate are formed by 36 unit cells like those described in [3]. The size of the structure is $1_{x}=21.259 \mathrm{~mm}$ in $\mathrm{x}$ direction, $\mathrm{l}_{\mathrm{y}}=17.272 \mathrm{~mm}$ in $\mathrm{y}$ direction and $\mathrm{l}_{\mathrm{z}}=7.366$ in $\mathrm{z}$ direction. 
In order to reduce the computational time and due to the symmetrical configuration, the whole configuration has been split and electromagnetic symmetry properties have been applied. The configuration can be seen in Fig. 1. (a).

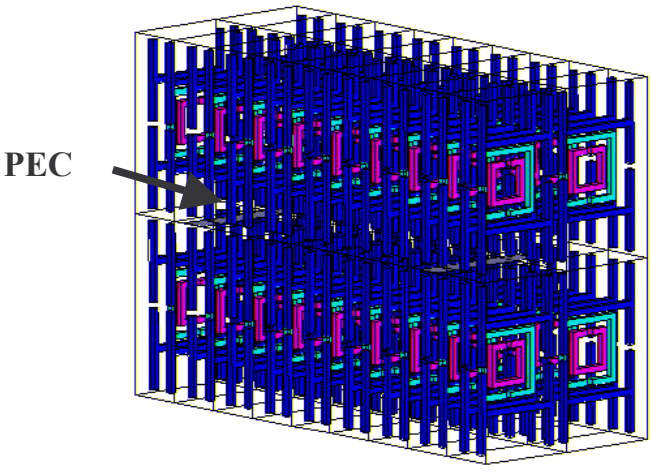

(a)

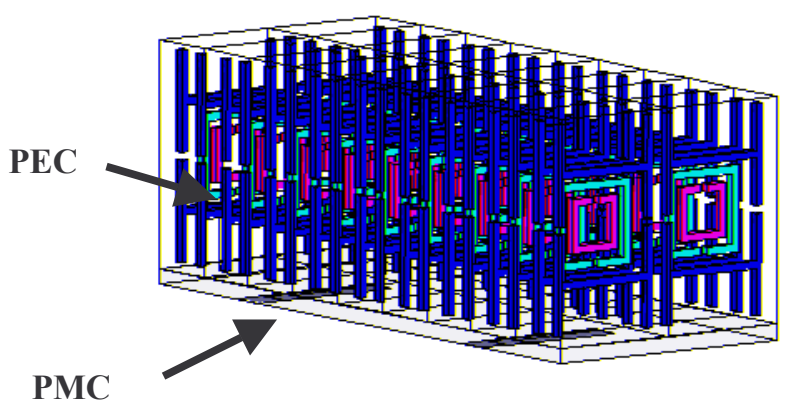

(b)

Fig. 1. Array configuration (a) One symmetry property (b) Two symmetry properties

Due to the complexity of the structure, two symmetry conditions have been defined: PEC (Perfect Electric Conductor) on the vertical face and PMC (Perfect Magnetic Conductor) on the horizontal face. This configuration can be seen in Fig. 1. (b). The dipoles have been placed in the middle of a dielectric substrate of $\varepsilon_{\mathrm{r}}=2.2$. This configuration allows to make a parametric study of the coupling changing the horizontal distance between dipoles. Besides, changing parametrically the thickness of the dielectric substrate together with the position of the dipoles, a study of the optimum distance from the dipoles to the superstrate and substrate can be fulfilled.

\section{Array configuration without losses}

Firstly, the array configuration with ideal materials, i.e., perfect conductor strips and dielectric without losses, have been analysed. The position of the dipoles has been varied in order to determine the radiation properties in each case. The results for the cases of $\lambda_{0} / 2$ and $\lambda_{0} / 4$ distance $\left(\lambda_{0}=\right.$ free space wavelength) between dipoles are shown in Fig. 2 because they are the most important in terms of coupling, radiation patterns and gain.

Analysing the radiation patterns (see Fig. 2), the endfire radiation is lower in Fig. 2 (a), however the directivity is also smaller. In the Fig. 2 (b), the endfire radiation obtained is higher but also higher directivity.

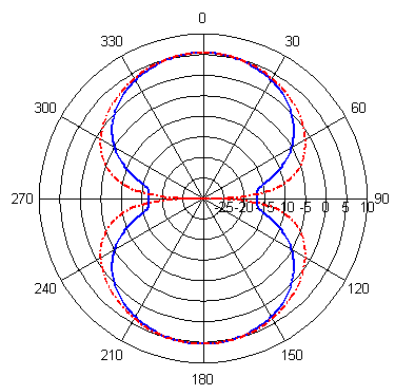

(a)

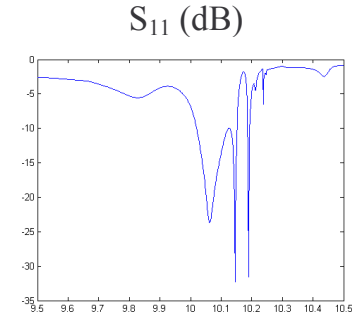

Distance $=\lambda / 2 \quad$ Resonant frequency $=9.88 \mathrm{GHz}$
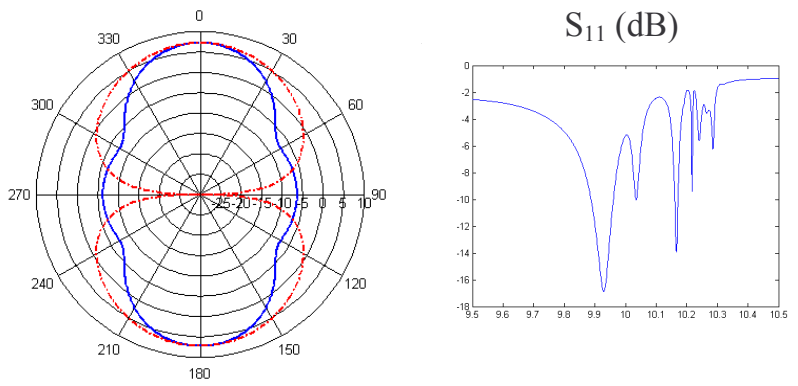

(b)

Fig. 2. Radiation patterns without losses for two distance between dipoles (a) $\lambda / 2$ and (b) $\lambda / 4$

Due to the electromagnetically symmetry property (PMC) applied in the plane of the dipoles, symmetrical radiation patterns are obtained. 
The coupling value depends on the power radiated by dipole antenna. As a result, the $S_{21}$ parameter does not give directly the desired coupling parameter, but a correction factor has to be applied as function of the radiated power to obtain the real coupling, given by equations (1) and (2). Taking into account the power radiated and reflected by each antenna and the power coupled between antennas, a more real coupling can be calculated, shown in Fig. 3.

$$
\begin{gathered}
P_{21}=P_{1}\left(1-\left|S_{11}\right|^{2}\right)\left|S_{21}\right|^{2}\left(1-\left|S_{22}\right|^{2}\right) \frac{P_{21}}{P_{1}}=\left|S_{21}^{\Gamma}\right|^{2} \\
\text { Coupling }(d B)=\left|S_{21}^{\Gamma}\right|^{2}=10 \log \left(\frac{\left|S_{21}\right|^{2}}{\left(1-\left|S_{11}\right|^{2}\right)\left(1-\left|S_{22}\right|^{2}\right)}\right)
\end{gathered}
$$

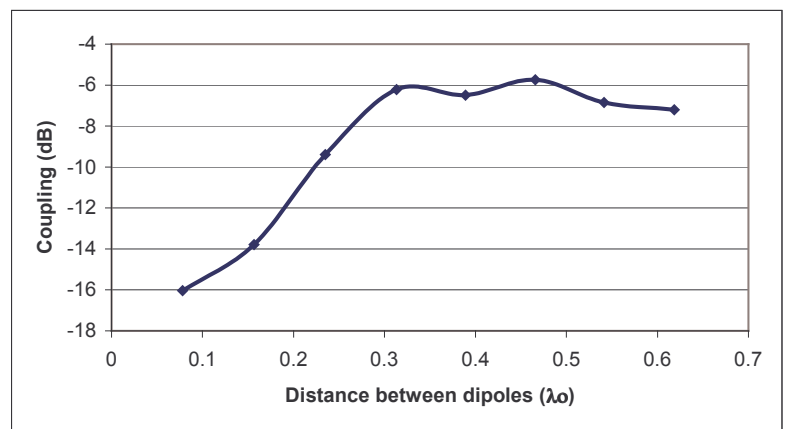

$\mathrm{P}_{21}=$ power in the antenna 2 due to the power radiated by the antenna 1

$\mathrm{P}_{1}=$ power in the antenna 1

Fig. 3. Corrected coupling between dipoles

As a conclusion, in the case of $\lambda_{0} / 2$, coupling between dipoles is maximum, however, side lobes in the radiation pattern are minimum. In the case of $\lambda_{0} / 4$, the coupling decreases and the gain is maximum.

\section{Array configuration with losses}

The next step to make the results closer to the reality is justo to study a more real configuration; i.e., losses in the dielectric material and copper strip are taken into account.

The material selected to create the unit cells is RT/duroid 5880, which is characterised by a dielectric constant of $\varepsilon_{\mathrm{r}}=2.2 \pm 0.02$ and a dissipation factor of $\tan \delta=0.0009$. Taking into account these losses, new simulations have been carried out. The radiation patterns obtained in both cases can be seen in Fig. 4.

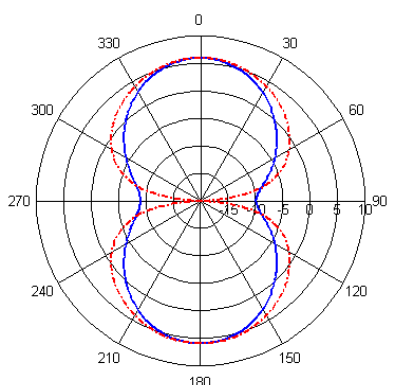

(a)

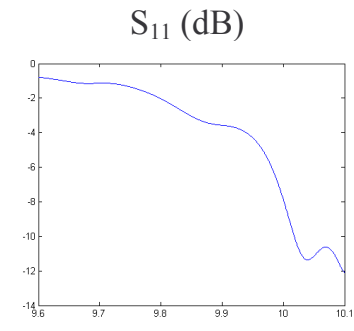

Distance $=\lambda / 2 \quad$ Resonant frequency $=9.80 \mathrm{GHz}$
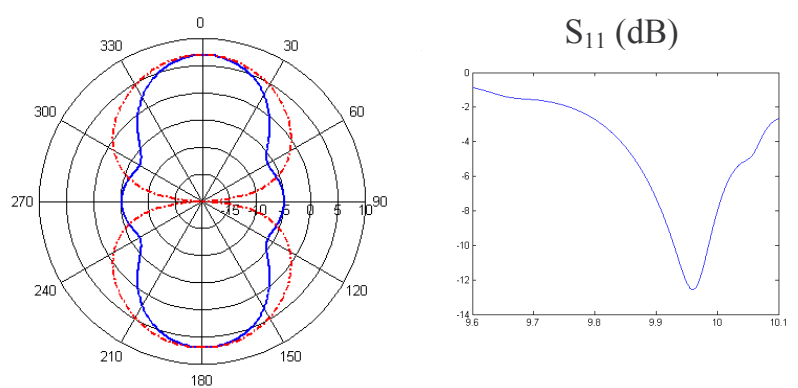

(b)

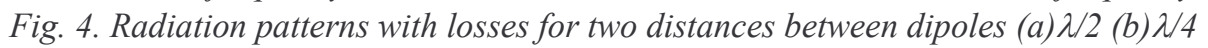

The radiation pattern shape is the same as it was expected. With a distance between dipoles of $\lambda_{0} / 2$, side lobes in the $\mathrm{H}$ plane (blue line) are minimized. However, in the case of $\lambda / 4$, directivity is maximum.

In order to check the effect of the material losses and the use of non-perfect metallic strips on the radiation properties, directivity, aperture efficiency, gain and radiation efficiency will be studied. Using the directivity, the radiated power and the incident power predicted by Ansoft HFSS, all parameters can be calculated. Results can be observed in Fig. 5. 


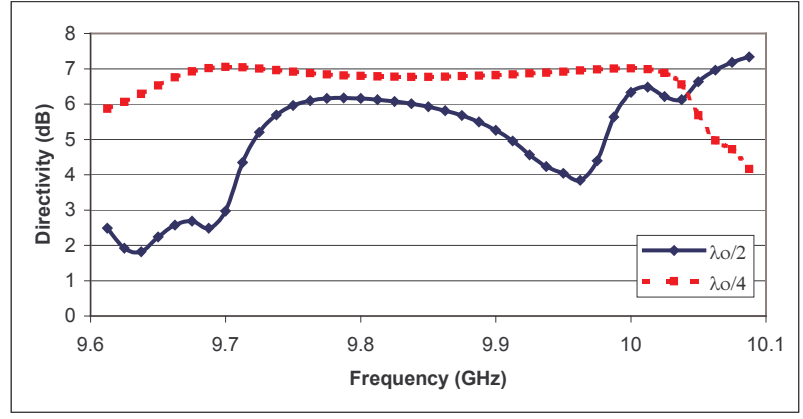

(a)

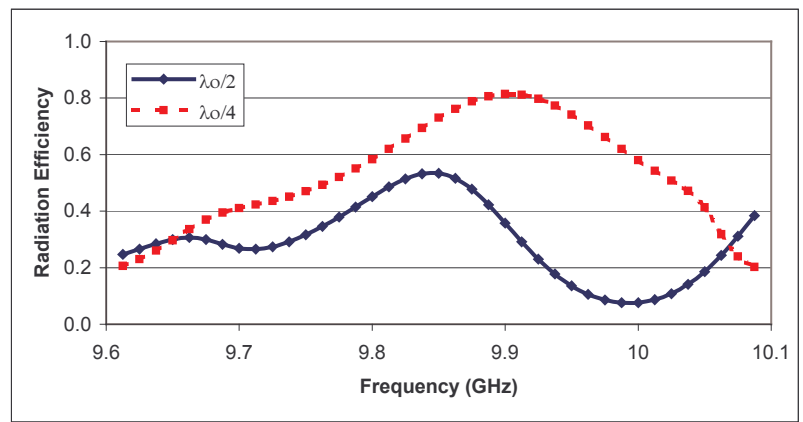

(c)

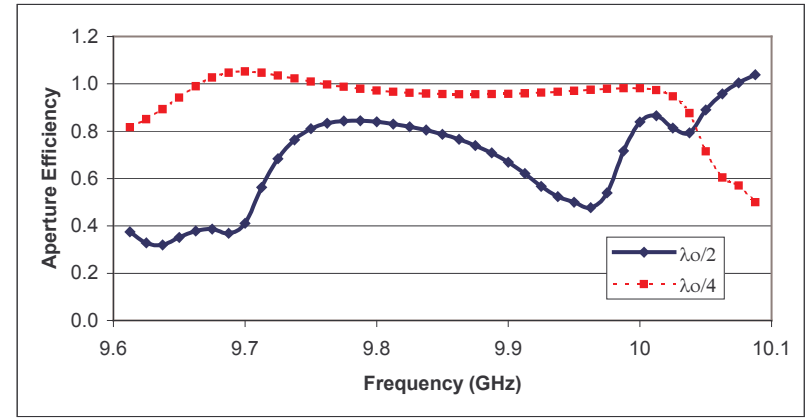

(b)

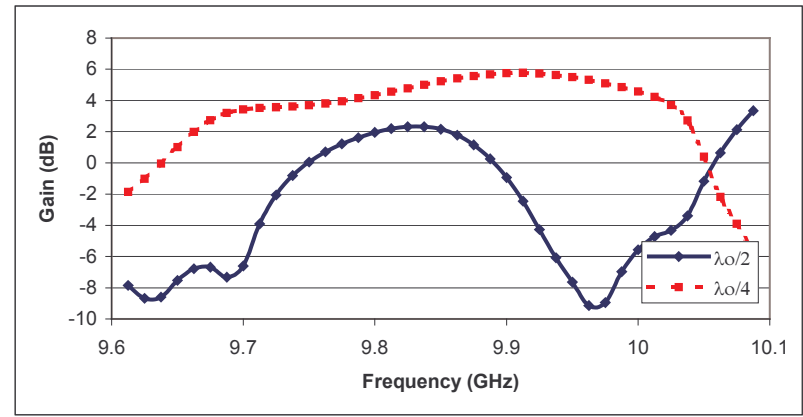

(d)

Fig. 5. Radiation parameters (a) Directivity (b) Aperture Efficiency (c) Radiation Efficiency and (d) Gain

In the case of a distance between dipoles of $\lambda_{0} / 4$, higher directivities in a wider bandwidth are obtained, with values around $7 \mathrm{~dB}$. As the coupling between dipoles is smaller, the radiation efficiency is higher, with values of 0.81 at $9.91 \mathrm{GHz}$. Taking into account the directivity, the aperture efficiency and the radiation efficiency, the gain can be calculated. Gains around $6 \mathrm{~dB}$ at $9.91 \mathrm{GHz}$ have been obtained. As a resonant structure, this configuration suffers from a reduced bandwidth operation.

\section{CONCLUSIONS}

In this paper efficient radiation systems formed by a LHM and a dipole antenna have been presented. A dipole array configuration, tuned at the resonant frequency of the LHM, has been inserted between a lefthanded superstrate and substrate. The radiation performances as directivity, gain, efficiency and radiation patterns have been analysed.

The radiation parameters have been calculated taking into account losses in the dielectric material and copper strips. It has been observed that the coupling between antennas has an important influence, having a larger efficiency when the coupling decreases.

\section{ACKNOWLEDGEMENTS}

The research presented in this paper has been financially supported by the Navarra Government under resolution 17/2004.

\section{REFERENCES}

[1] V.G. Veselago, "The electrodynamics of substances with simultaneously negative values of $\varepsilon$ and $\mu$ ", Sov. Phys. Usp. 10, 509 (1968).

[2] R. W. Ziolkowski, "Design, Fabrication, and Testing of Double Negative Metamaterials", IEEE Transactions on Antennas and Propagation, Vol. 51, No. 7, July 2003.

[3] E. Sáenz, R. Gonzalo, I. Ederra, P. De Maagt, "High Efficient Dipole Antennas by Using Left-Handed Superstrates", $13^{\text {th }}$ International Symposium on Antennas JINA 2004. 
